\title{
Universidas transdisciplinaria en el marco de la sociedad del conocimiento. Tensionses discursivas en la Universidas de Chile
}

\author{
Transdisciplinary University in the Framework of the Knowledge \\ Society. Discursive Tensions Within Universidad de Chile
}

\author{
Andree Henríquez Aravena ${ }^{1,2}$, Marco Billi ${ }^{13,4}$, Valentina Faúndez ${ }^{3}$, \\ Julio Labraña ${ }^{1,4,5}$, Ignacio Neira ${ }^{1,3}$, Anahí Urquiza ${ }^{1,3,4}$ \\ ${ }^{1}$ Núcleo de Investigación-Acción en Interdisciplina y Transdisciplina \\ para la Educación Superior (NITES), Universidad de Chile. \\ 2 Observatorio de Innovación, Facultad de Economía y Negocios, Universidad de Chile. \\ ${ }^{3}$ Núcleo de Estudios Sistémicos Transdisciplinarios, \\ Facultad de Ciencias Sociales, Universidad de Chile. \\ ${ }^{4}$ Centro de Ciencia del Clima y la Resiliencia CR2 (FONDAP № 1511009). \\ ${ }^{5}$ Centro de Políticas Comparadas de Educación, Universidad Diego Portales.
}

\section{Resumen}

En el marco de las transformaciones sociales contemporáneas, y del nuevo rol adoptado por las universidades, la transdisciplina ha ido ganando un reconocimiento creciente como a sroximación sara comprender fenómenos que no sueden ser abordados por el modelo tradicional de especialización disciplinar, como el cambio climático, la desigualdad o, recientemente, las pandemias. Sin embargo, existe escaso estudio de experiencias específicas que ayuden a entender qué implica, qué finalidad tiene y cómo puede lograrse una mayor focalización transdisciplinaria en las universidades. Para reducir esta brecha, este artículo analiza los resultados de un diálogo sarticipativo realizado al interior de la Universidas de Chile, examinando distintas narrativas respecto de la emergencia, oportunidades y obstáculos de la transdisciplina; los que, a su vez, dicen relación con las dinámicas históricas, organizacionales y culturales propias de la trayectoria adoptada por esta institución, y la educación superior en general, en el país: i) el compromiso social, inscrito en la tradición y misión académica de la universidad pública; ii) una promesa de novedad, innovación y transformación del quehacer científico y académico, asociada al modelo emergente de universidad emprendedora; y iii) la creciente demanda avanzada para una reforma profunda del sistema de educación superior en el país.

Palabras clave: transdisciplina, sociedad del conocimiento, Universidad de Chile, educación superior, innovación.

\section{Correspondencia a:}

Andree Henríquez Aravena

Observatorio de Innovación, Facultad de Economía y Negocios, Univessidad de Chile Diagonal Paraguay 265. Piso 14, Of. 1405, Santiago.

andree.henriquez@uchile.cl

Este artículo fue financiado por la Vicerrectoría de Investigación y Desarrollo, Universidad de Chile; Proyecto PFUE UCH-1799l, Ministerio de Educación; Fondecyt $\mathrm{n}^{\circ}$ 11200429; FONDAP $\mathrm{n}^{\circ}$ 15110009. Además, se agradece a los académicos y funcionarios que participaron del workshop ilustrado en el artículo.

(C) $2021 \mathrm{PEL}$, http://www.pensamientoeducativo.org - http://www.pel.cl 


\section{Abstract}

Amidst contemporary social transformations, including the new role adopted by universities, transdisciplinarity has been gaining increasing recognition as an approach to understanding phenomena that cannot be addressed by the traditional model of disciplinary specialization: these include climate change, inequality, or, more recently, pandemics. However, there is little study of specific experiences that help to understand the implications, purpose, and challenges of adopting a transdisciplinary approach in universities. In order to reduce this gap, this article analyses the results of a participatory dialogue carried out in Universidad de Chile, examining different narratives regarding the emergence of transdisciplinarity and the associated opportunities and obstacles. These, in turn, relate to the historical, organizational, and cultural dynamics of the trajectory adopted by this institution, and the higher education sector in general, in the country: i) the social commitment, inscribed in the tradition and academic mission of the public university; ii) a promise of novelty, innovation, and transformation of scientific and academic work, associated with the emerging model of an entrepreneurial university; and, iii) the growing demand for a profound reform of the higher education system in the country.

Keywords: transdiscipline, knowledge society, University of Chile, higher education, innovation.

\section{Introducción}

En las últimas décadas, las universidades y centros de investigación, históricamente los actores más importantes en el proceso de generación y diseminación del conocimiento científico han enfrentado significativas transformaciones producto de diversas tendencias globales (Etzkowitz, Webster, Gebhardt, \& Terra, 2000). En este contexto, la transdisciplina (TD) ha ganado progresivamente un espacio en el mundo académico, cuestionando los límites tradicionales de la ciencia, trasgrediendo el foco disciplinar e integrando actores no científicos a la generación de conocimiento (Stokols et al., 2003; Wang, Aenis, \& Siew, 2019). Su atractivo ha estado en subrayar el carácter multidimensional y sistémico de los problemas contemporáneos, presionando hacia un diálogo colaborativo, con base en preguntas que, por su envergadura, requieren superar el modelo de ciencia newtoniana (Stokols et al., 2003), impulsando un cambio desde una investigación para la sociedad hacia una con la sociedad (Knapp, Reid, Fernández-Giménez, Klein, \& Galvin, 2019).

El surgimiento y desarrollo de la TD debe entenderse vinculado al concepto de complejidad (Rosenfield, 1992), que caracteriza fenómenos donde muchos procesos y componentes interactúan con la capacidad de generar una nueva calidad de comportamiento colectivo, cuyas manifestaciones son la formación espontánea y emergente de estructuras temporales, espaciales o funcionales distintivas, para cuya comprensión el conocimiento científico debe buscar nuevos caminos (Cilliers, 1992; Daudé et al., 2009; Goldstein, 2013). Esto ha significado un giro sustancial respecto de los modelos tradicionales de producción y reproducción científica, donde la definición, representación y solución de los problemas de investigación se abordan dentro de los límites disciplinares (Scholz, 2017).

Para lograr lo anterior, la TD refuerza su orientación al problema de investigación cruzando los bordes disciplinares, innovando en metodologías para dar cuenta de la complejidad, y fomentando la colaboración entre la comunidad científica y extra-científica (Russell, Wickson, \& Carew 2008). En línea con lo anterior, especialmente desde la mitad de la década de 1990, proliferan diversos intentos para incorporar enfoques transdisciplinarios 
en las universidades, como una respuesta a la creciente complejidad y urgencia de los desafíos de investigación y educación superior, específicamente en los ámbitos de la salud y de la sustentabilidad medioambiental (Aneas, 2015; Dlouha, Huisingh, \& Barton, 2013; Krainer \& Winiwarter, 2016; Mauser et al., 2013; Scholz, 2017).

Si bien la producción de conocimiento ha tenido un rol fundamental para la supervivencia y perpetuación del ser humano desde su origen (David \& Foray, 2002), los últimos 30 años han traído transformaciones radicales, donde la habilidad para crear, distribuir y explotar el conocimiento se ha constituido como un elemento crucial para mejorar el bienestar de las personas (Holm-nielsen \& Agapitova, 2002) y como principal impulsor del desarrollo de los países (Carayannis, 2013; Leydesdorff, 1996).

A su vez, es preciso considerar que la importancia productiva del conocimiento es parte de un proceso globalizante de mayor envergadura, comenzado en Estados Unidos con la promoción de la investigación aplicada en el contexto de la competencia de la Guerra Fría y, sucesivamente, impulsado por múltiples organismos internacionales desde la década de 1990 (Slaughter \& Leslie, 1997). Esta nueva sociedad del conocimiento posindustrial (de Weert, 1999), se caracteriza por una aceleración en la producción de conocimiento, el surgimiento de nuevas tecnologías (Powell \& Snellman, 2004), la centralidad de la innovación (David \& Foray, 2002), y una creciente relación universidad-industria para la generación y explotación de la investigación, desarrollo e innovación ( $+\mathrm{D}+\mathrm{D}+\mathrm{i})$ como recurso económico (Lundvall, 1996). En este sentido, la TD emerge en un contexto de creciente transformación global, donde el conocimiento alcanza un estatus central para la sociedad.

En esta dirección, la declaración de Bolonia indica que el conocimiento se estaría convirtiendo en un "factor irremplazable para el crecimiento social y humano" en un mundo crecientemente interconectado (Bologna Process, 1999, p.1). Asimismo, la Estrategia de Lisboa auspiciada por la Unión Europea (Lisbon European Council, 2000, p.1), busca convertir al continente en "la economía basada en el conocimiento más competitiva y dinámica del mundo, capaz de sustentar el desarrollo económico con más y mejores trabajos y una mayor cohesión social". Transformaciones comparables se pueden encontrar en el contexto asiático, particularmente en Japón, que posterior a la Segunda Guerra Mundial fue transitando desde un sistema de educación superior enfocado en la formación de funcionarios gubernamentales a uno centrado en las relaciones entre industria y academia como motor de crecimiento económico del país (Etzkowitz et al., 2000).

Por otra parte, para América Latina esto ha significado una alteración del rol de la educación superior que, hasta mediados de 1970, era concebida como expresión de la voluntad del Estado, implicando que sus actividades estuviesen vinculadas, principalmente, al cumplimiento de objetivos de interés nacional (Levy, 1986; Etzkowitz et al., 2000). Aunque muchas universidades públicas sigan manteniendo este rol, se observa un cambio profundo hacia convertirse en un instrumento para la formación de capital humano y generación de conocimiento, conduciendo a una alteración profunda en su identidad (Mollis, 2006; Tünnermann, 2003).

Más específicamente, el sistema de educación superior chileno ha experimentado transformaciones radicales desde 1980, de la mano de cambios en el financiamiento estatal (Brunner, 1986), el establecimiento de sistemas de aseguramiento de la calidad (Bernasconi y Rojas, 2004; Marínez, Labrańa, y Matus, 2019) y el condicionamiento de la entrega de recursos a resultados de docencia e investigación (Labrańa y Rodríguez, 2017). Ulteriores reformas persiguieron el fin declarado de impulsar una transición hacia una economía del conocimiento, estableciendo diversos fondos para incentivar a las universidades a incorporar enfoques interdisciplinarios, transdisciplinarios y un mayor compromiso social en sus actividades (Ministerio de Educación, 1997; Salazar y Leihy, 2013).

Pese a lo anterior, la mayor parte de la literatura especializada opta por argumentar sobre la deseabilidad de estos cambios o por criticar sus premisas ideológicas (Brunner, 2009; Brunner, Labraña, Ganga, y Rodríguez-Ponce, 2019), persistiendo importantes brechas en la comprensión de cómo estas transformaciones son percibidas, valoradas e integradas por parte de académicos y directivos. A su vez, la escasez de trabajos que analicen las posibilidades, 
desafíos y experiencias específicas dirigidas a reorientar a las universidades hacia una mayor transdisciplinariedad ha impedido evaluar adecuadamente las oportunidades y obstáculos que enfrentan en la práctica los procesos de transformación organizacional, o de prestar la debida atención a las expectativas ancladas en la TD en los contextos de educación superior. Esto último adquiere especial relevancia, considerando las tensiones discursivas que constitutivamente lleva asociada la TD y que, como propone Nicolescu (2010), nos muestran un campo todavía en construcción, pero a la vez clave para el desarrollo futuro de los países (Jahn, Bergmann, \& Keil, 2012).

Este artículo busca colmar esta brecha mediante el análisis de las narrativas y tensiones discursivas en la discusión sobre TD y los propósitos de ciencia, educación y extensión en la Universidad de Chile, examinando su interpretación y las oportunidades y obstáculos que implican para la transformación de los sistemas de gestión de conocimiento en Chile. Con este fin, se situarán dichas narrativas y tensiones en el contexto de las dinámicas históricas, organizacionales y culturales que han marcado el desarrollo del país.

Siguiendo esta línea, el artículo se estructura en cinco secciones. Se comienza describiendo cómo la política pública chilena de las últimas décadas ha buscado fortalecer la investigación básica y aplicada, con el propósito expreso de fomentar una economía basada en el conocimiento, y cómo la Universidad de Chile, una de las principales instituciones de investigación del país, ha incorporado estas demandas entre sus lineamientos. Sucesivamente, se presenta la metodología de investigación (estudio de caso), el mecanismo de generación de información (metálogo) y el modo de análisis (análisis del discurso). A continuación, se ilustran los resultados del estudio, enfocándose en hacer emerger los principales polos discursivos que articulan las narrativas sobre TD en el contexto de la Universidad de Chile, para luego discutir su significado en el marco de transformaciones organizacionales, nacionales y globales. El artículo finaliza con un resumen, futuras líneas de investigación y sugerencias de política.

\section{La transición de Chile hacia la sociedad del conocimiento y la Universidad de Chile}

Durante las últimas décadas, diferentes gobiernos chilenos han enfatizado la importancia de las universidades como un elemento clave para la competitividad del país. Esta nueva orientación, distinta a la clásica comprensión de las instituciones culturales, dominante entre los siglos XIX y XX (Labraña, 2018; Ruiz-Schneider, 2010), ha quedado reflejada en múltiples iniciativas destinadas a incentivar la creación de vínculos entre universidades, organizaciones de la sociedad civil y empresas privadas. De acuerdo con la Organización para la Cooperación y Desarrollo Económicos (OCDE, 2017), en Chile la inversión en ciencia y tecnología creció un 13\%, solo entre 2005 y 2017. Gran parte de estos nuevos recursos han tenido por objetivo incentivar la producción de conocimiento en las instituciones de educación superior, en asociación con industrias y comunidades (FONDEF, 2018). Un ejemplo de esta tendencia es el Programa Regional de Investigación Científica, fondo creado en 2010 para mejorar las capacidades de ciencia, tecnología e innovación en regiones del país. Su principal foco ha estado en la creación y fortalecimiento de centros científicos y tecnológicos de carácter asociativo, con una interrelación activa con diferentes actores territoriales, en un contexto interdisciplinario (Programa Regional de Investigación Científica y Tecnológica, 2018).

Esta transformación en las prioridades de la política pública respecto de la educación superior ha resultado en nuevos desafíos para las universidades chilenas, especialmente en términos de investigación; esto refleja las tendencias globales que promueven una asignación de recursos públicos y privados, condicionada a la creación de vínculos con actores no universitarios (Clark, 2004; Slaughter \& Leslie, 1997, 2001; Slaughter \& Rhoades, 2004), y la creciente valorización de la idea de universidad como una organización activa socialmente (Brunner et al., 2019; Krücken, 2003; Ramírez, 2006). Lo anterior demanda a estas instituciones avanzar en procesos de reforma interna para poder fortalecer sus capacidades interdisciplinarias (Bernasconi, 2006; Pineda, 2014, 2015). 
El caso de las universidades estatales resulta especialmente interesante. En contraste con las universidades privadas, que por su estructura diversificada de ingresos (Brunner, Labraña, y Álvarez, 2019) enfrentan menores trabas burocráticas para introducir reformas con criterios de mercado (Bernasconi, 2011), las instituciones estatales deben expandir y diferenciar la plataforma de investigación dentro de sus unidades académicas; aumentar la productividad para responder a retos privados y estatales; fortalecer las relaciones entre sus facultades y departamentos, y competir dentro y fuera del país, sin abandonar sus misiones tradicionales asociadas al bien común, so pena de perder su legitimidad entre estudiantes, académicos y funcionarios, afectando incluso su imagen pública frente a actores económicos y políticos (Matus y Núñez, 2002; Grandón y Vargas, 2002; Koch \& Vanderstraeten, 2018).

Como se espera mostrar en este trabajo, esta tensión entre desafíos globales y respuestas locales a la demanda social desde un enfoque transdisciplinar se refleja en los profundos y recientes procesos de reforma de la Universidad de Chile, evidenciados en sus planes de desarrollo institucional de la última década. Siguiendo la senda trazada por el Proyecto de Desarrollo Institucional del año 2006, que estableció entre sus objetivos estratégicos avanzar hacia una formación e investigación interdisciplinaria y transdisciplinar, el Plan de Desarrollo Institucional 2017-2026 (PDI 2017-2026) definió "el fomento de los necesarios diálogos inter y transdisciplinarios tanto al interior de la Universidad como con actores externos" (Universidad de Chile, 2006) como uno de los valores centrales de la institución y sus diferentes unidades.

El PDI 2017-2026 establece la necesidad de la universidad de adaptarse a la sociedad del conocimiento, incorporando especialmente abordajes transdiciplinarios para lidiar con problemas sociales, científicos y culturales. Asimismo, plantea como desafío principal de la investigación universitaria "crear condiciones para que se produzcan interacciones entre las ciencias, las humanidades y las artes con la sociedad -problemáticas país y desafíos globales relevantes-a través de iniciativas inter y transdisciplinarias que permitan el mejoramiento de la actividad institucional, el fortalecimiento del Sistema de Educación Superior Estatal y aporten al desarrollo de políticas públicas" (Universidad de Chile, 2018, p. 31). En esta estrategia de investigación, la colaboración transdisciplinaria aparece como un criterio central para evaluar la pertinencia de las actividades de los académicos, tal como se evidencia en las distintas iniciativas de financiamiento internas a la Universidad de Chile. Por ejemplo, el Concurso Generación de Redes de Investigación Transdisciplinaria para Desafíos Globales, creado por la Vicerrectoría de Investigación y Desarrollo (VID) en 2018, apunta a fomentar el establecimiento de redes de investigación transdisciplinarias entre académicos de la Universidad de Chile, otras instituciones nacionales e internacionales de educación superior y organizaciones de la sociedad civil. Asimismo, los proyectos de internacionalización de la investigación y doctorados, UCH-1566 y UCH-1866, han puesto el mismo énfasis.

Más allá de evaluar el éxito o fracaso de estas iniciativas - cuestión que escapa a los fines de este trabajo-resulta del mayor interés analizar en qué medida estas nuevas expectativas sobre la naturaleza de la producción y utilización del conocimiento son incorporadas a la reflexión de los miembros de la Universidad de Chile. Como veremos a continuación, este proceso no refleja de manera automática las definiciones de TD presentes en la literatura especializada, así como tampoco las intenciones de los legisladores de reorientar a las universidades hacia una economía basada en el conocimiento, sino que combina estas expectativas con énfasis propios de la trayectoria institucional de la Universidad de Chile, resultando de este modo en una variedad idiosincrática de la idea misma de TD.

\section{Metodología}

Para reconstruir las tensiones discursivas sobre TD y los propósitos de la ciencia, el artículo emplea una aproximación de investigación transdiciplinaria, denominada metálogo. Esta noción fue acuñada por el antropólogo Gregory Bateson como una forma de diálogo en la cual los participantes discuten simultáneamente de un objeto o problema (comunicación) y de cómo se comprende este objeto o problema (metacomunicación). 
Un metálogo es una conversación sobre algún teme poblemático. La conversación tiene que ser tal, que no sólo los participantes discutan efectivamente el poblema sino que la estructura de la conversación en su totalidad sea también pertinente al mismo tema (Bateson, 1972, p. 17).

Siguiendo esta idea, se utilizó una versión de metálogo construida específicamente para las ciencias sociales (Ufquiza, Amigo, Billi, Bandấo, y Morales, 2018) como una técnica transdisciplinaria orientada a curmpliz simultáneamente dos funciones. En un primer nivel, el metálogo fomenta la reflexividad de los participantes y la co-construcción de un boundary object: un objeto simbolizado y consensuado (usualmente en la forma de un acta, un documento de trabajo o similar) que pueda luego servir como referencia común para las diferentes perspectivas involucradas, promoviendo su futura colaboración y coordinación alrededor de los problemas y conceptos articulados en el boundary object (Star \& Griesemer, 1989). En un segundo nivel, el metálogo moviliza significantes y significados en relación con el objeto del diálogo, haciendo emerger consensos solapados y eventuales tensiones discursivas, que pueden visibilizarse sometiendo el piopio metálogo a un análisis de discurso.

La aplicación del metálogo al caso en examen consistió en los siguientes pasos (figura 1):

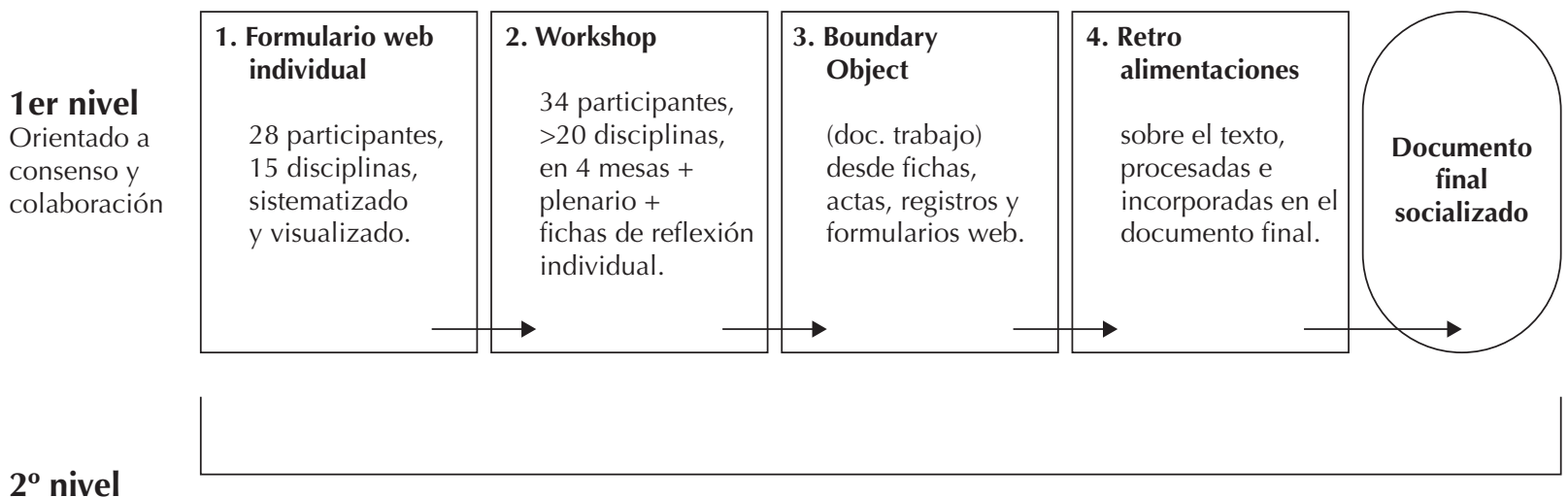

Orientado a

discuiso y

Análisis cualitativo del discurso

tensiones

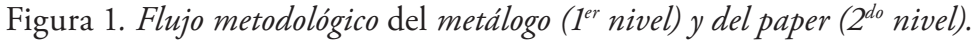

1. Se aplicó un formulario web individual, recolectando los aportes de 28 participantes provenientes de 15 disciplinas, sobe la definición de $\mathbf{~} f D$, las condiciones para aplicarla exitosamente en investigación y docencia, y las experiencias existentes al respecto en la Universidad de Chile. Los participantes fueron seleccionados por bola de nieve (Berg \& Lene 2017) en función de su experiencia en el tema dentro de la universidad. Las respuestas fueron sistematizadas en la forma de nubes de palabras y mapas conceptuales, para utilizarse en la fase siguiente.

2. Se realizó un Workshop de investigación interdisciplinaria y transdiciplinaria durante el cual, estimulados por las respuestas sistematizadas en la fase 1 y con la facilitación de profesionales capacitados, los participantes dialogaron y reflexionaron alrededor de las comprensiones e imaginarios sobre $\mathrm{TD}$, así como sobre las oportunidades, desafís, estrategias y requerimientos implicados para ponerla en práctica al interior de la Universidad de Chile. En el Workshop participaron 34 asistentes de más de 20 disciplinas, inicialmente separados en cuatro mesas de trabajo, y luego reunificados en un plenario. En paralelo, se invitó a los participantes a llenar una ficha de reflexión personal. 
3. Fichas, actas, registros audiovisuales y formularios web fueron sucesivamente analizados, conduciendo a la elaboración de un boundary object que buscaba reflejar el resultado consensuado de la co-construcción realizada por medio del metálogo. Este fue sometido nuevamente a discusión entre los participantes, quienes dejaron comentarios, observaciones y sugerencias en el texto, los cuales fueron utilizados para refinar el documento, para posteriormente ser socializado al interior de la universidad y entre sus principales referentes institucionales.

Posteriormente, la información obtenida en las fases anteriores fue procesada por medio del análisis del discurso, específicamente mediante la técnica de análisis de contenido guiado inductivamente. Esta permite identificar patrones y temas implícitos en el discurso de manera inductiva, con el objetivo de caracterizar el fenómeno en estudio de manera integral (Berg \& Lune, 2017). En el caso de esta investigación, la finalidad del uso de esta técnica fue evidenciar narrativas y divergencias respecto de la comprensión de la TD, así como las oportunidades y desafíos que su ejecución involucra.

\section{Resultados}

El estudio vislumbra que las narrativas sobre TD relatadas dentro de la Universidad de Chile se organizan alrededor de tres polos articuladores: i) una promesa de novedad, innovación y transformación del quehacer científico y académico, ii) el compromiso social, inscrito en la tradición y misión académica de la Universidad de Chile, y iii) la oportunidad y necesidad de una reforma, tanto de la universidad como de su entorno (figura 2). Cada uno de estos, tal como argumentaremos en la sección siguiente, cataliza las narrativas reproducidas en el espacio propio de la Universidad de Chile, como también los imaginarios y discursos movilizados en el contexto relevante de aquella, tanto a nivel local y nacional, como global.

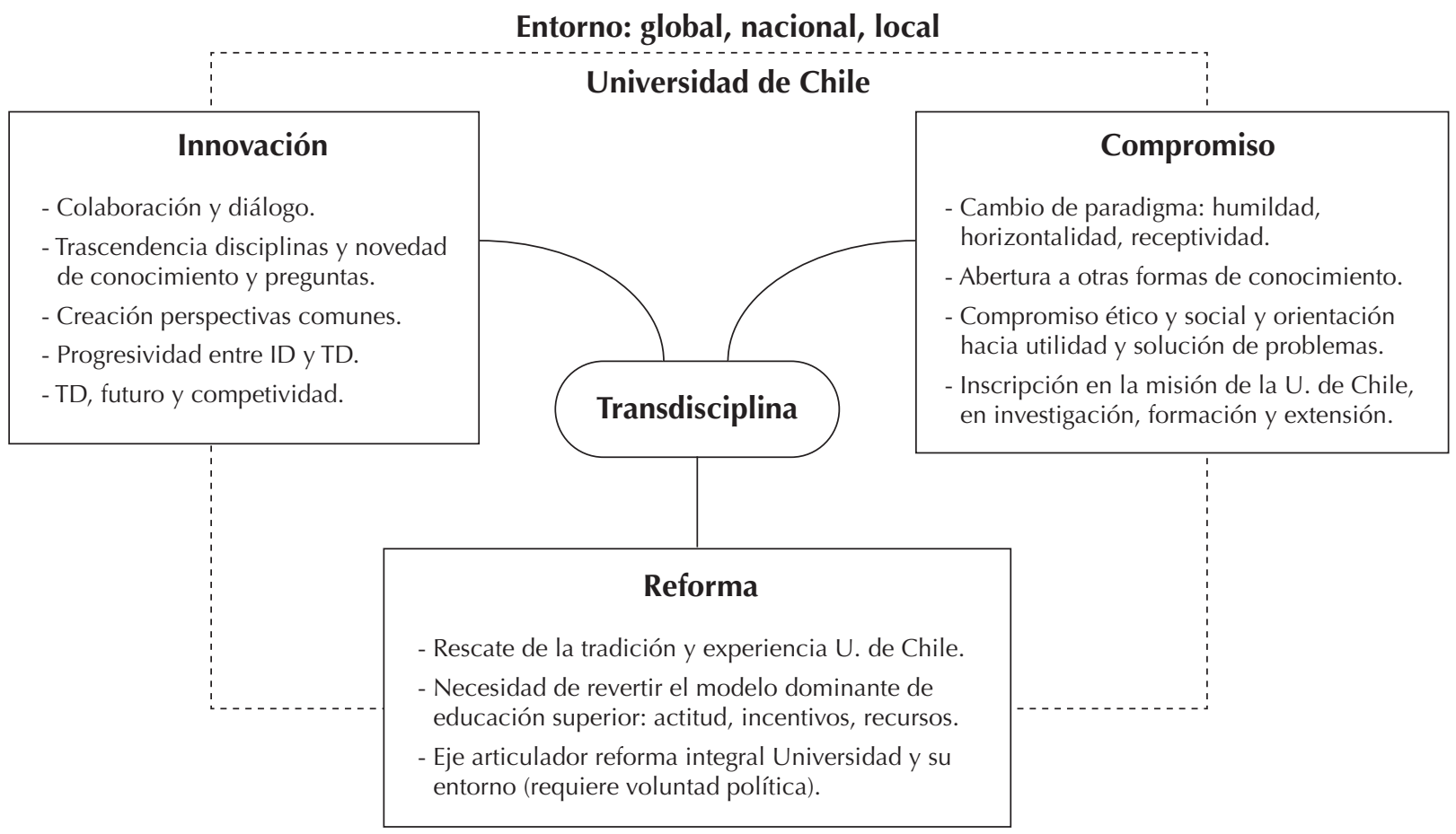

Figura 2. Resultados, organizados según polo discursivo. 
A continuación, se ahondará dentro de cada polo, describiendo las principales narkativas que llevan asociadas. Para ejemplificarlas, se mostrarán al gunos fragmentos del discurso de los actores, identificados según la instancia de levantamiento del discuroo (fortulario, workshop, ficha) y el ámbito disciplinario al cual se adscriben.

\section{Transolisciplina e ínovación acadénica}

En la base de la $\mathrm{TD}$ se encontraría, de acuerdo con los participantes, un comptomiso por superar la percibida fragmentación que afecta a modalidades disciplinarias o multidisciplinarias de conocimiento, proveyendo una plataforma más adecuada para abordar la complejidad. Se discute que la Tí buscaría fomentar la colaboración

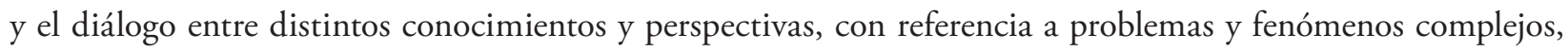
generando comprensiones más integradas y completas sobre ellos.

"[Transdisciplina significa] abordar un problema o ámbito con mirada común, no con las miradas que parceladamente aporta cada disciplina" (Cuestionario, ciencias de la salud).

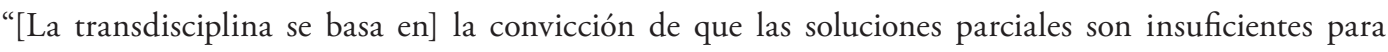
resolver problemas complejos, y es necesario pensar en conjuinto para adaptar respuestas más adecuadas" (Cuestionario, ciencias biológicas y ambientales).

Pese a la importancia que los conceptos de colabofoción y diálogo revisten para esta perspectiva, estos son raramente indicados como elementos propiamente distintivos de la TD, y parecen posicionarse como elementos de continuidad entre esta y la interdisciplina. La característica que tiende a señalare como marormente distintiva de la $\mathrm{CD}$ es más bien la novedad del conocimiento que produce. Según seńalan los informantes, aunque la interdisciplina también requiere de colaboración y diálogo, y puede fomentar interpetaciones y aprendizajes recípocos entre disciplinas, se limitaría por lo general a un a combinación o sumatoria de perspectivas disciplinarias,

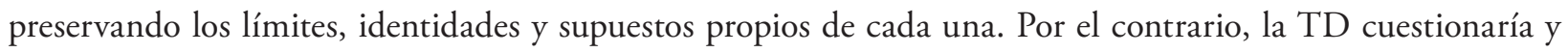
trascendería dichos límites, identidades y supuestos, generando conocimientos nuevos en función de pioblemas también nuevos, esto es, no heredados de las disciplinas de origen.

"La característica principal de la investigación transdisciplinar cadica en la disposición a cuestionar los límites del conocimiento adquirido y a incorporar los saberes de las diferentes áreas buscando construir un nuevo esquema explicativo" (Cuestionario, ciencias sociales).

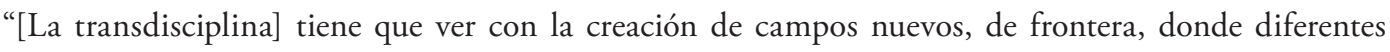
epistemologías, metodologías y saberes se combinan y fusionan para generar una nueva forma de indagación, híbrida por definición" (Cuestionario, ingeniería y arquitectura).

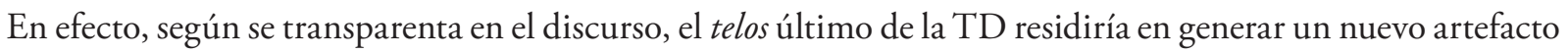

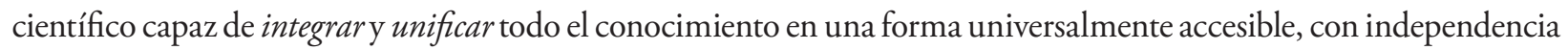
de la pocedencia disciplinaria. Por lo tanto, el éxito de la $\mathrm{TD}$ se mediría, al menos en parte, por su capacidad

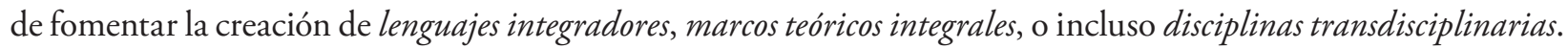

"Transdisciplina ocurre cuando el trabajo conjunto de dos o más disciplinas conlleva la creación de una nueva disciplina, que aborda poblemas que van más allá del alcance inicial de las disciplinas constituyentes"(Cuestionario, ciencias físico-matemáticas).

"Busca la formación de una nueva disciplina a la frontera entre dos o más disciplinas, con base en un enfoque holístico (como puede ser el marxismo, los estudios culturales o de género, por ejemplo). Busca la unificación del conocimiento más allá de las disciplinas" (Cuestionario, ciencias sociales). 
De esa manera, para diversos informantes, existiría una relación de progresividad entre interdisciplina y TD, presentándose esta última como un paso sucesivo, más radical y ambicioso, en el intento de trascender la división disciplinaria para crear conocimientos y paradigmas nuevos y más integrales.

"Multidisciplinariedad, interdisciplinariedad y transdisciplinariedad son etapas sucesivas (o progresivas) en la superación del encierro disciplinario y en la integración de distintas formas de conocimiento" (Cuestionario, ciencias sociales).

"Multidisciplina, interdisciplina, transdisciplina: pasos en la capacidad de contextualizar, integrar, crear, problematizar, conocimientos disciplinados" (Fichas, ciencias de la salud).

En línea con lo anterior, los participantes describen la apuesta de la Universidad de Chile por una perspectiva transdisciplinaria. Por un lado, como una oportuna e incluso necesaria respuesta frente a las actuales transformaciones que experimentan los sistemas de conocimiento y la sociedad, incluyendo la emergencia de problemas y fenómenos más complejos y multidimensionales. Por el otro, como una oportunidad estratégica para posicionar a la universidad en la vanguardia en el escenario nacional e internacional, visibilizando y valorizando la relevancia de su oferta formativa, haciéndola más competitiva respecto de otras instituciones de educación superior.

"Es la nueva forma de hacer universidad" (Workshop, ciencias de la salud).

"Nos situaría en la vanguardia para conocimiento necesario para la sociedad del futuro" (Ficha, ciencias sociales).

Sin embargo, no existe una completa consonancia con relación al alcance de la transformación que la TD ofrece, respecto de la manera tradicional de pensar la investigación y la universidad. En efecto, para algunos esta debería comprenderse como un abordaje de carácter residual, es decir, justificable solo frente a situaciones particularmente complejas, imposible de abordarse empleando las tradicionales herramientas disponibles a nivel disciplinario.

"Si la complejidad del problema no lo requiere, no es necesaria la transdisciplina" (Workshop, ciencias biológicas y ambientales).

Otros informantes, no obstante, enfatizan la necesidad de posicionar la TD de manera más bien transversal respecto de todo el quehacer científico y académico.

"Debe entenderse como un esfuerzo permanente, no solo frente a objetos críticos o situaciones o eventos complejos" (Ficha, ciencias sociales).

\section{Transdisciplina y compromiso social}

Si el primer polo discursivo hacía énfasis, principalmente, en el potencial creativo e integrador de la TD como una nueva forma de hacer ciencia y producir conocimiento, el segundo enfatiza más bien la capacidad transformadora de la ciencia, cuestionando su posición tradicional con respecto de otras esferas sociales. En el discurso, se enfatiza a la TD como una respuesta a la forma establecida de delimitar y validar problemas y conocimientos científicos. Esto conduciría a la TD, en la opinión de los participantes, hacia el fomento de la consciencia respecto de los límites epistemológicos de la ciencia y la necesidad de abrazar, por consecuencia, una actitud más humilde, horizontal y receptiva respecto de la diversidad.

"Una contribución no jerarquizada, horizontal de varias disciplinas" (Cuestionario, ciencias biológicas y ambientales). 
Este proceso, sostienen algunos, debiese completarse mediante la apertura significativa de la investigación transdisciplinaria hacia otras de formas de conocimiento tradicionales, locales o no formales, ajenas a aquellas usualmente movilizadas en ámbitos académicos. La articulación de estas formas de conocimiento sería una de las más importantes contribuciones de la TD.

"La transdisciplina es un proceso de co-construcción de conocimiento entre diferentes agentes transformadores: entre estos, expertos, comunidad, agentes privados y públicos” (Ficha, ciencias sociales).

"Integrando distintos saberes, no solo disciplinares, sino también políticos, cotidianos, religiosos, etc. Va más allá de la disciplina" (Cuestionario, ciencias sociales).

Otros participantes discrepan de estas posiciones, subrayando el dilema de conciliar dicha apertura a lo no científico, con la preservación del valor propiamente epistémico de la ciencia, así como de los desafíos adicionales que aquello representaría respecto de las dificultades propiamente científicas comportadas por la TD. Muchos advierten la necesidad de diferenciar la TD, entendida por ellos como operación interna a la ciencia, respecto de los esfuerzos por construir una más estrecha interfaz entre ciencia, política y sociedad.

"Se confunde la co-construcción de conocimiento científico con la co-construcción de conocimiento para la toma de decisiones, donde efectivamente el conocimiento local es clave" (Cuestionario, ciencias sociales).

"[La interfaz] implica el conocimiento popular/local y apunta en nuevos mares epistemológicos, los cuales no son imprescindibles en la interdisciplina" (Ficha, ciencias sociales).

En contraste, goza de mucho mayor consenso la interpretación de la TD como una respuesta a la forma establecida de justificar la relevancia de problemas y campos de investigación. De acuerdo con los informantes, mientras el conocimiento disciplinario comenzaría desde inquietudes y problemas definidos como relevantes a partir de la intertextualidad de la propia comunicación científica, la TD incorporaría una predilección por problemas socialmente relevantes y útiles.

"La transdisciplina en cambio es una aproximación a la investigación que genera un nuevo conocimiento útil para la sociedad" (Cuestionario, ciencias biológicas y ambientales).

"[La transdisciplina opera] creando, definiendo, un objeto no solo de estudio sino de intervención (acción) o interacción entre los conocimientos y el problema" (Ficha, ciencias sociales).

Concorde con esta conceptualización, la transdisciplina no debería limitarse a producir conocimiento; por el contrario, le correspondería asumir un compromiso ético y social, lo cual la haría necesaria no solo en presencia de problemas complejos, sino también frente a situaciones o dilemas que sean valórica o políticamente controversiales.

"Cuando algo afecta a las personas (no decisiones operativas), tenemos que recurrir a la transdisciplina" (Workshop, funcionario).

"[La transdisciplina] es una palanca de Arquímedes para llegar a pensar en grande la innovación, al servicio de transformar la sociedad" (Fichas, ciencias sociales).

Esta interpretación sostiene a su vez una forma alternativa de justificar y valorizar la apuesta de la Universidad de Chile por una perspectiva transdisciplinaria. En adición a enfatizar, como se describió en el apartado precedente, la capacidad de la transdisciplina de proyectar la organización y la innovación hacia el futuro, los participantes también anclan la TD con el pasado, la tradición y la misión de la Universidad. En este sentido, para los participantes, la identidad de la Universidad de Chile se asienta en su carácter de institución pública, con un 
fuerte compromiso social y político. La TD, en consecuencia, se vislumbra como una herramienta clave para la transformación de la sociedad y la solución de los problemas contemporáneos que afectan a la gente, y se convierte por lo tanto en una pieza esencial para cumplir con su compromiso público.

"[La transdisciplina] permite responder y concretar la función social de la universidad y su compromiso no solo con la ciencia sino con la sociedad chilena y el resto del mundo" (Fichas, ciencias sociales).

"Tiene que ver con el relato de la misión institucional de la universidad, la necesidad de posicionarse en un espacio, somos una universidad pública" (Workshop, ingeniería y arquitectura).

Para ello, no se considera suficiente adoptar la TD como un enfoque para la investigación, sino que aquella debiese jugar un rol fundamental en orientar las estrategias formativas y de extensión de la universidad. Desarrollar formas de docencia transdisciplinaria, en particular, se advierte como una necesidad impelente tanto frente a la creciente complejidad de los problemas que los profesionales del futuro enfrentarán como para asegurar que aquellos adopten integralmente ese mismo compromiso social que se adscribe a la universidad.

"Los problemas actuales son inter y transdisciplinarios, y la docencia es tendencialmente monodisciplinaria: hay un vacio" (Ficha, ingeniería y arquitectura).

"Es importante formar a profesionales con la capacidad de hacerse cargo de los problemas país y con herramientas para trabajar con otros" (Ficha, ciencias sociales).

\section{Transdisciplina y reforma institucional}

Un tercero y último polo discursivo se funda en las consecuencias y desafíos asociados a la adopción de un enfoque transdisciplinario. En línea con el énfasis puesto en relevar el anclaje de la TD en la tradición de la Universidad de Chile, los participantes destacan como fundamental visualizar las experiencias existentes dentro y alrededor de la organización, y crear redes entre los académicos y sus unidades.

"Hacer un catastro de [...] formas de organización local que los académicos han creado para dar cabida a la transdisciplina" (Ficha, ciencias sociales).

"Antes que nada, saber quiénes somos y qué hacemos. Hay un terrible desconocimiento interno sobre el potencial que tiene la U en esta temática" (Ficha, ciencias biológicas y ambientales).

Al mismo tiempo, los informantes subrayan que la TD encuentra cierta rigidez institucional, debido a la resistencia que ejerce el modelo dominante en el cual se encontraría todavía inserta la educación superior en el país. Esto, a pesar de que la TD parece estar anclada en la misión institucional de la universidad.

La superación de dicho modelo, el que se denomina según el caso positivismo, neoliberalismo, productivismo o extractivismo académico, queda entonces estrechamente vinculada al uso de enfoques transdisciplinares.

"[Existe] una tendencia historia en la $\mathrm{U}[\ldots]$ a una formación académica monodisciplinar [...] asociada a un modelo de sociedad mercantil" (Ficha, ingeniería y arquitectura).

"[Se requiere] romper una lógica de generación de conocimiento individual y solo disciplinar" (Ficha, ciencias sociales). 
La TD, en este sentido, requeriría un cambio en las maneras en las que tradicionalmente ha operado la universidad. Este cambio debería partir de los propios académicos: en efecto, tal como se mencionó anteriormente, los participantes enfatizan cómo la TD requiere poner en juego competencias y actitudes humanas asociadas con valores como humildad, receptividad y horizontalidad, que no están necesariamente presentes en el perfil establecido del personal universitario.

"La receptividad a diferentes perspectivas, una actitud integradora e innovadora para expandir los límites conocidos y probados, además de rigor y creatividad para que esa innovación haga sentido y tenga coherencia" (Ficha, ingeniería y arquitectura).

"La participación de sujetos abiertos al diálogo y a la cooperación genuina, con la capacidad de desbordar los límites conceptuales de su disciplina” (Cuestionario, ciencias sociales).

Además de resistencias culturales al cambio, los participantes identifican como un obstáculo esencial para la TD la falta de reconocimiento de las actividades realizadas tanto en términos de carrera como de las horas de dedicación que aquellas requieren a los académicos. Se necesitaría, argumentan, proveer nuevos incentivos y un grado suficiente de protección al docente e investigador para que salgan de sus fronteras disciplinarias.

"Lo más eficiente es quedarse en lo monodisciplinar. Hay que generar incentivos distintos al productivismo" (Ficha, ciencias biológicas y ambientales).

"La jornada, reconocer la jornada. Mientras la transdisciplina no entre en la jornada, que es la cancha chica de cada uno, no va a funcionar" (Workshop, funcionario).

Junto con la disposición de los individuos, los incentivos correctos y el tiempo laboral, el cambio requeriría también, según los informantes, de recursos adecuados, ya sea en términos de infraestructuras, espacios de encuentro o financiamientos dedicados a proyectos transdisciplinarios.

"Espacios formativos que apunten a la colaboración, trabajo en equipo interdisciplinario y la co-creación de conocimiento" (Cuestionario, ciencias biológicas y ambientales).

"Se requiere que la $\mathrm{U}$ obtenga fondos que garanticen independencia para estos esfuerzos" (Ficha, ciencias de la salud).

En este sentido, los participantes concuerdan que adoptar la TD no puede limitarse a incorporar formas alternativas de hacer ciencia, docencia o extensión; más bien, aquello debiese volverse la inspiración para una reforma institucional, que involucre todas las áreas de la universidad, y fundada en un fuerte compromiso por parte de las autoridades universitarias.

"La transdisciplina cambia a toda la universidad. Por tanto, cuenta no como otra actividad, sino que podría ser el núcleo de una gran reforma" (Ficha, ciencias sociales).

En efecto, justamente por la radicalidad de los cambios que la TD requiere, y porque aquella es tan dependiente de la existencia de una seria y constante voluntad política, lograr una completa e integral adopción de este principio solo será posible, según los participantes, a condición de que los propios interesados logren organizarse para hacerse escuchar y elevar presiones y requerimientos hasta hacer de la TD una prioridad estatal.

"El problema es la universidad, pero hay problemas estructurales más allá, las primeras correcciones fueron hechas por FONDAP [Fondo de Financiamiento de Centros de Investigación en Áreas Prioritarias]" (Workshop, ingeniería y arquitectura). 


\section{Discusión y conclusiones}

Los resultados confirman la hipótesis inicial de este artículo: si bien las narrativas movilizadas al interior de la Universidad de Chile con respecto a la TD rescatan, como es esperable, elementos usualmente asociados con el concepto dentro del debate internacional, la configuración específica que adoptan dentro de la construcción discursiva de la institución es un ensamblaje idiosincrático de los elementos puestos a disposición dentro de distintas tradiciones dominantes en la literatura especializada de TD. Así, tal como estudios anteriores han sugerido (Marginson, 2004), la apropiación del concepto queda influenciada por dinámicas y referentes semánticos tanto globales, como nacionales, e incluso propios de la trayectoria histórica singular del contexto en estudio.

En efecto, como mostraremos a continuación, los tres polos discursivos identificados conectan la TD con referentes semánticos-ideológicos propios de la trayectoria histórica de la Universidad de Chile.

Más precisamente, los primeros dos polos refieren a expectativas y descripciones propias de dos modelos alternativos de educación superior: por un lado, se sitúan en lo que Burton Clark (1998, 2004) llamaría universidad emprendedora, esto es, una institución capaz de responder a las demandas de los diferentes actores ubicados en su entorno y, de esta manera, contribuir al progreso de la sociedad toda (véase también Slaughter \& Leslie, 1997). Este modelo ha ido ganando importancia a nivel global en los últimos ańos, en el marco de cambios en los fines atribuidos a la investigación y docencia universitarias producto de la emergencia de la sociedad del conocimiento (Steinbicker, 2011). Asimismo, se ha ido consolidando también a nivel nacional, gracias a políticas de regulación y financiamiento, especialmente dominantes desde la segunda mitad de la década de 1990, que asignan especial importancia a la capacidad de las instituciones de convertir su investigación y docencia en actividades con relevancia práctica (Brunner, 2015; Labraña y Rodríguez, 2017).

Sin embargo, el modelo de universidad emprendedora ha sufrido críticas debido a su tendencia a desnaturalizar el rol público de la universidad en favor de una racionalidad economicista e individualista, centrada en la persecución de objetivos relacionados con la acumulación de prestigio y capital, sin mayor atención a su compromiso público tradicional (Peters, 1992; Giroux, 2014; Olssen, 2015). En esta línea, la universidad emprendedora queda contrapuesta al tradicional modelo de universidad pública históricamente vigente en Chile (Mellafe, Rebolledo, y Cárdenas 1992; Serrano, 1994; Ruiz-Schneider, 2010). Al respecto, debe notarse que, aunque en las últimas décadas el modelo de universidad pública tradicional ha entrado a competir con el de universidad emprendedora, aquel mantiene todavía su vigencia en el marco de una institucionalidad nacional e internacional que promueve la reforma social por la vía de la educación (Schofer \& Meyer, 2005). Se destaca, a este respecto, el surgimiento de fuertes llamados, tanto a nivel global como local, a repensar la estructura y misión de las universidades en el marco de la urgencia y complejidad asociadas a los contemporáneos problemas ambientales, y la importancia de la investigación y educación transdisciplinaria en el marco de los esfuerzos para poner en acto paradigmas de desarrollo sostenible (Labrańa et al., 2019; O’Brien et al., 2013; Yarime et al., 2012).

La TD recoge el desafío del modelo de universidad emprendedora en la medida en que, tal como se ha observado anteriormente, el potencial innovador de la TD y su orientación a proveer respuestas más integrales a problemas complejos queda discursivamente asociado a expectativas de competitividad y posicionamiento estratégico de la universidad dentro del panorama nacional e internacional de educación superior. De esa manera, abrazar la TD se presenta como una respuesta posible frente a las crecientes presiones ejercidas sobre la institución, tanto por los legisladores como por su propio entorno socioeconómico: es decir, para promover su conversión en motor de desarrollo científico, tecnológico y económico dentro de una economía crecientemente basada en el conocimiento.

Por otro lado, el discurso enfatiza también las aspiraciones transformativas de la TD y su compromiso ético, político y social, lo que se hace explícitamente como una autodescripción de la tradición y misión institucional de la Universidad de Chile, en tanto entidad pública dedicada a velar por el bien común, la equidad y cohesión 
social, y la crítica y movilización activa con respecto del quehacer político del país. Es decir, en consonancia con el modelo tradicional de universidad pública. En efecto, la TD se representa no solo como la lógica continuación de los valores y prioridades propias de la institución, sino también como una necesidad para asegurar que aquella pueda cumplir con su cometido y una herramienta para potenciar su capacidad de intervención y cambio social, sin alterar los elementos fundamentales de su propia autocomprensión.

De esta manera, ambos modelos de educación superior (universidad pública y universidad emprendedora) encuentran un terreno común precisamente en la TD, que se convierte tanto en una prioridad como en un medio para promover simultáneamente ambos conjuntos de finalidades y, a la vez, proveer una reconciliación discursiva para las expectativas y prioridades asociadas a cada uno de los dos modelos. La noción de TD opera, de esta manera, como un concepto viajero (Said, 1983): un concepto que circula entre instituciones, adquiriendo nuevos sentidos de acuerdo con sus contextos de enunciación, en un proceso constante de interpretación y reinterpretación que establece las bases para la consolidación de sistemas de gestión del conocimiento a nivel local, nacional y regional.

El tercer polo discursivo identificado desarrolla la forma de una demanda concertada para una reforma organizacional e institucional. En efecto, ambos modelos de universidad (universidad emprendedora y universidad púbica con compromiso social) concuerdan en criticar y rechazar el paradigma que domina actualmente la configuración de la educación superior chilena: ambos destacan las rigideces, los límites y los condicionamientos que aquel supone para el actuar académico, y ambos se encuentran unidos por el anhelo de transformar profundamente los sistemas de conocimiento en el país. Las demandas de reforma de la educación superior en Chile han adquirido particular intensidad en el contexto chileno después del movimiento estudiantil de los años 2011 y 2012, mediante sus reclamos por una educación pública, gratuita y de calidad. Este inició un proceso de discusión entre los distintos actores del sistema acerca de los objetivos de las instituciones de educación superior, en especial en entidades estatales como la Universidad de Chile, sin que hasta la fecha se vislumbre un claro consenso en esta materia (Guzmán-Valenzuela, Barnett, \& Labrańa, 2019; Bernasconi, 2014). Por el contrario, este aparece como uno de los principales temas de controversia en el marco de las protestas que han azotado el país desde octubre de 2019.

Por cierto, esta síntesis de perspectivas operada por la TD no anula la profunda diferencia (teórica, práctica e ideológica) que divide los dos modelos de universidad pública y universidad emprendedora. En efecto, es esperable que pese al común acuerdo por rechazar la institucionalidad vigente y la TD, estas diferencias adquieran mayor importancia a la hora de proponer metas y caminos específicos para un atto de transición hacia una universidad más transdisciplinaria. Esto queda patente en nuestro análisis, al considerar la profunda heterogeneidad de perspectivas respecto de cómo llevar adelante la TD, qué barreras enfrenta, y qué instrumentos la debiesen apoyar. Ahí se hacen visibles divergencias que, si no son cuidadosamente reflexionadas y procesadas por mecanismos dialógicos idóneos (como aquellos fomentados por instancias como el metálogo que aquí se analizó), pueden terminar minando todo intento de avanzar hacia la TD en la universidad.

Para cerrar, vale destacar las líneas de estudio futuras y recomendación de política pública que se abren a partir de esta investigación.

Respecto de lo primero, como hemos mostrado, el uso del concepto de TD en la Universidgd de Chile emerge a partir de procesos de traducción mediados por la trayectoria de la organización, las características del contexto nacional y las definiciones de la literatura especializada sobre el tema. Considerado lo anterior, futuros estudios podrían analizar si acaso en otros sistemas de educación superior, históricamente influenciados por el modelo de la universidad socialmente comprometida, como la mayoría de los países de la región latinoamericana (Mollis, 2006; Pineda, 2015), estos procesos se dan de manera similar o si acaso el peso de las tradiciones intelectuales de cada país juega un rol preponderante en la operacionalización del sentido de la TD. 
En esta línea, cabe destacar que, a partir del análisis, se identifican numerosos contenidos discursivos cuya asociación con la idea de TD generó controversias y divisiones entre los mismos participantes. Estos incluyen, entre otros: si el tránsito hacia un enfoque transdisciplinario puede resolverse en el seno de la misma ciencia o bien requiere necesariamente trascender los límites entre esta y otras formas de conocimiento; si la transformación de la forma de hacer investigación y docencia debiese verse como medio para un fin (por ejemplo, incrementar la innovación o el valor agregado para la sociedad) o como un fin en sí mismo, y si, más en general, el impulso hacia la TD implica fomentar una transformación más amplia y radical en la propia forma de concebir la ciencia y su relación con la sociedad. Ya que estas controversias dividen también a la literatura especializada, sería interesante contrastar la forma en la que estas se procesan en el caso chileno -plagado por las tensiones propias de un sistema con un alto grado de privatización- con las trayectorias que aquellas toman en otros contextos sociohistórico-geográficos.

En esta misma línea, cabe notar que la TD también inspira miradas críticas. Estas no surgieron de forma explícita en el material que analizamos, posiblemente debido a un sesgo de autoselección de quienes participaron en la discusión. Sin embargo, sí es posible reconocer ciertas resistencias, sobre todo frente a las interpretaciones más radicales y transformadoras de TD, y el intento de algunos informantes por poner límites al alcance, tipo de problemas y ámbitos de aplicabilidad específica de la TD. Futuras investigaciones deberían indagar de manera más atenta estas reservas latentes, así como a su reticencia a hacerse explícitas, con el fin de dar luces sobre la dimensión política-controversial de la TD e informar mejor el diseño de iniciativas orientadas a promover su aceptación en la praxis académica.

En términos de política pública, el artículo destaca la relevancia de la TD como eje de una reforma institucional y organizacional que permita responder a las presiones a las que se encuentra crecientemente expuesta la universidad, en el marco de la predominancia de una economía fundada en el conocimiento, sin renunciar a la tradicional misión social propia de las instituciones universitarias públicas. La Universidad de Chile se encuentra en una posición privilegiada para liderar una reforma de este tipo, tanto en el país como en la región, en virtud del acervo cultural con el que cuenta en la materia y de su actual compromiso por desarrollar experiencias pioneras al respecto. Para aquello, asignar a la TD un papel protagónico dentro de la estrategia de desarrollo de la institución puede ayudar a asegurarle la visibilidad, integralidad y la continuidad de compromiso que necesita para desarrollar todo su potencial transformador.

En este marco, es preciso resaltar que instancias de reflexión transdisciplinarias, como la relatada en este artículo, cumplen una función crucial por lo menos en dos niveles. Por un lado, son esenciales para construir una referencia común y un grado suficiente de consenso sobre el cual fundar la colaboración y coordinación necesarias para institucionalizar la TD al interior de la Universidad de Chile. Por el otro, propician una oportunidad para hacer emerger, contrastar y reflexionar distintas perspectivas, imaginarios y narrativas sobre un tema de gran contingencia para la actualidad del país, como la reforma de los sistemas de educación superior. En este punto, la técnica del metálogo ha mostrado ser una metodología particularmente adecuada, tanto para promover el diálogo y la construcción de acuerdos como en su calidad técnica de investigación dirigida a visibilizar e investigar narrativas y meta-narrativas alrededor de objetos sociales disputados (Urquiza et al., 2018).

El artículo original fue recibido el 9 de diciembre de 2019 El artículo revisado fue recibido el 8 de junio de 2020 El artículo fue aceptado el 1 de julio de 2020 


\section{Referencias}

Aneas, A. (2015). Transdisciplinary technology education: a characterisation and some ideas for implementation in the university. Studies in Higher Eduction, 40(9), 1715-1728. https://doi.org/10.1080/03075079.2014.899341

Bateson, G. (1972). Pasos hacia una ecología de la mente. Una aproximación revolucionaria hacia la autocomprensión del hombre. Buenos Aires, Argentina: Editorial Lohlé-Lumen.

Berg, B. L. \& Lune, H. (2017). Qualitative research methods for the social sciences. Boston, MA: Pearson.

Bernasconi, A. (2006). Breaking the institutional mold: Faculty in the transformation of Chilean higher education from state to market. En H. Dieter-Mayer \& B. Rowan (Eds.), The New Institutionalism in Education (pp. 33-50). Albany, NY: State University of New York Press.

Bernasconi, A. (2011). Caminos público y privado hacia la Universidad de Investigación de Catesoría Mundial: el caso de Chile. En P. G. Altbach (Ed.), The road to academic excellence: The making of world class research universities (pp. 229-260). Washington, D.C.: The World Bank.

Bernasconi, A. (2014). Policy path dependence of a research agenda: the case of Chile in the aftermath of the student revolt of 2011. Studies in Higher Education, 39(8), 1405-1416. https://doi.org/10.1080/03075079.2014.950448

Bernasconi, A. y Rojas, F. (2004). Informe sobre la educación superior en Chile: 1980-2003. Santiago, Chile: Editorial Universitaria.

Bologna Process. (1999). The Bologna declaration of 19 June 1999: Joint declaration of the European Ministers of Education. Recuperado de https://www.eurashe.eu/library/bologna_1999_bologna-declaration-pdf/

Brunner, J. J. (1986). Informe sobre la Educación Superior en Chile. Santiago, Chile: FLACSO.

Brunner, J. J. (2009). Sociology of higher education in the international, regional, and local contexts. Dados-Revista De Ciencias Sociais, 52(3), 621-658. https://doi.org/10.1590/S0011-52582009000300003

Brunner, J. J. (2015). Medio siglo de transformaciones de la educación superior chilena: Un estado del arte. En A. Bernasconi (Ed.), La educación superior de Chile: Transformación, desarrollo y crisis (pp. 21-108). Santiago, Chile: Ediciones Universidad Católica de Chile.

Brunner, J. J., Labraña, J., y Álvarez, J. (2019). Inversión de la sociedad en educación superior. Enfoque de Políticas ESTP, 2.

Brunner, J. J., Labraña, J., Ganga, F., y Rodríguez-Ponce, E. (2019). Circulación y recepción de la teoría del “academic casitalism" en América Latina. Archivos Analiticos de Politicas Educativas, 27(79), 1-32. https://doi.org/10.14507/epaa.27.4368

Carayannis, E. G. (2013). Encyclopedia of Creativity, Invention, Innovation, and Entrepreneurship. Nueva York, NY: Springer Link. https://doi.org/10.1007/978-1-4614-3858-8

Cilliers, P. (1992). What Can We Learn from a Theory of Complexity? Emergence, 2(1), 23-33. https://doi.org/10.1207/S15327000EM0201_03

Clark, B. R. (1998). Creating entrepreneurial universities: Organizational pathways of transformation. Oxford, NY: Pergamon Press.

Clark, B. R. (2004). Sustaining change in universities: Continuities in case studies and concepts. Nueva York, NY: The Society for Research into Higher Eductition \& Open University Press.

Daudé, E., Provitolo, D., Dubos-Paillard, E., Gaillard, D., Eliot, E., Langlois, P., Propeck, E., \& Saint-Gérand, T. (2009). Spatial risks and complex systems: methodological perspectives. En M. Aziz-Alaoui y C. Bertelle (Eds.), From System Complexity to Emergent Properties (pp. 165-178). Berlín, Alemania: Springer.

David, P. A. \& Foray, D. (2002). An introduction to the economy of the knowleyge society. International Soctal Science Journal, 54(171), 9-23. https://doi.org/10.1111/1468-2451.00355

de Weert, E. (1999). Contours of the Emeryent Knowleyge Society: Theoreticyl Debate and Implications for Higher Education Research. Higher Education, 38(1), 49-69. https://doi.org/10.1023/A:1003756429869

Dlouha, J., Huisingh, D., \& Barton, A. (2013). Learning networks in higher education: universities in search of making effective regional impacts. Journal of Cleaner Production, 49, 5-10. https://doi.org/10.1016/j.jclepro.2013.01.034

Etzkowitz, H., Webster, A., Gebhardt, C., \& Cantisano, B. (2000). The future of the university and the university of the future: evolution of ivory tower to entrepreneurial paradigm. Research Policy, 29, 313-330. 
Fondo de Fomento al Desarrollo Científico y Tecnológico (FONDEF). (2018). Ciencia y desarrollo para Chile: FONDEF 25 años. Santiago, Chile: CONICYT.

Giroux, H. A. (2014). Neoliberalism's war on higher education. Chicago, IL: Haymarket Books.

Goldstein, J. (2013). Emergence: A Construct amid a Thicket of Conceptual Snares. Emergence, 2(1), 5-22. https://doi.org/10.1207/S15327000EM0201_02

Grandón, E. y Vargas, G. (2002). Movilidad social intergeneracional: una medida de la contribución social de las universidades. El caso de los titulados de la Universidad del Bío-Bío, Chile. En Centro Interuniversitario de Desarrollo (CINDA) (Ed.), Seguimiento de egresados e inserción laboral: Experiencias universitarias (pp. 275-294). Santiago, Chile: CINDA.

Guzmán-Valenzuela, C., Barnett, R., \& Labraña, J. (2019). Consensus and dissensus: changing perceptions of the public dimension of universities in a marketised environment. Journal of Higher Education Policy and Management, 42(1), 49-66. https://doi.org/10.1080/1360080X.2019.1658850

Holm-nielsen \& Agapitova, N. (2002). Chile - Science, Technology and Innovation. Washington, D.C: World Bank.

Jahn, T. Bergmann, M., \& Keil, F. (2012). Transdisciplinarity: Between mainstreaming and marginalization. Ecological Economics, 79, 1-10. https://doi.org/10.1016/j.ecolecon.2012.04.017

Knapp, C. N., Reid, R. S., Fernández-Giménez, M. E., Klein, J. A., \& Galvin, K. A. (2019). Placing transdisciplinarity in context: A review of approaches to connect scholars, society and action. Sustainability (Switzerland), 11(18), 1-25. https://doi.org/10.3390/su11184899

Koch, T. F. \& Vanderstraeten, R. (2018). Internationalizing a national scientific community? Changes in publication and citation practicesinChile, 1976-2015.CurrentSociology, 67(5),723-741.https://doi.org/10.1177/0011392118807514

Krainer, L. \& Winiwarter, V. (2016). Universities as Actors in Transformative Science. Consequences for Assessing Transdisciplinary Research Quality. GAIA-Ecological Perspectives for Science and Society, 25(2), 110-117.

Krücken, G. (2003). Learning the 'new, new thing': On the role of path dependency in university structures. Higher Education, 46(3), 315-339. https://doi.org/10.1023/A:1025344413682

Labraña, J. (2018). The cultural understanding of the universities and its relationship with dissemination media: An analysis of the semantic changes in the concept of the Chilean university between 1990 and 2010 (tesis doctoral). Witten, Germany: Fakultät für Kulturreflexion, Universität Witten/Herdecke.

Labraña, J. y Rodríguez, J. (2017). Estado y universidad en Chile: problemas de distinción en torno a su función pública. Pensamiento Educativo. Revista de Investigación Educacional Latinoamericana, 54(1), 1-19. https://doi.org/10.7764/PEL.54.1.2017.7

Labrańa, J., Amigo, C., Cortés, J., Gómez, E., Moreno, J., y Muńoz, M. C. (2019). Hacia una educación ambiental para una sociedad compleja. Un análisis desde la teoría de sistemas sociales. Revista Mad - Universidad de Chile, (39), 13-45. https://doi.org/10.5354/0718-0527.2019.53283

Levy, D. C. (1986). Higher education and the state in Latin America: Private challenges to public dominance. Chicago, IL: The University of Chicago Press.

Leydesdorff, L. (1996). Emergence of a Triple Helix of university-industry—government relations. Science and Public Policy, (23)5, 279-286. https://doi.org/10.1093/spp/23.5.279

Lisbon European Council. (2000). Presidency council. Recuperado de https://www.europarl.europa.eu/summits/lis1_en.htm

Lundvall, B. (1996). The Social Dimension of The Learning Economy. DRUID WORKING PAPER, 96(1). Recuperado de https://ideas.repec.org/p/aal/abbswp/96-1.html

Marginson, S. (2004). Competition and Markets in Higher Education: a 'glonacal' analysis. Policy Futures in Education, 2(2), 175-244. https://doi.org/10.2304/pfie.2004.2.2.2

Maríńez, C., Labraña, J. y Matus, T. (2019). Coordinación sistémica en la esfera pública. Observando la conversión de la experticia científica en confianza pública desde el modelo sistémico funcional y el modelo pragmático formal. Cinta de Moebio: Revista Electrónica de Epistemología de Ciencias Sociales, 65, 209-226. Recuperado de https://cintademoebio.uchile.cl/index.php/CDM/article/view/54034/57949

Matus, S. y Núńez, E. (2002). Fortalecimiento de redes y vinculación con ex alumnos de la Universidad de Talca. Experiencia y propuesta de modelo. En Centro Interuniversitario de Desarrollo (CINDA) (Ed.), Seguimiento de egresados e inserción laboral: Experiencias universitarias (pp. 245-257). Santiago, Chile: CINDA. 
Mauser, W., Klepper, G., Rice, M., Schmalzbauer, B. S., Hackmann, H., Leemans, R., \& Moore, H. (2013). Transdisciplinary global change research: The co-creation of knowledge for sustainability. Current Opinion in Environmental Sustainability, 5(3-4), 420-431. https://doi.org/10.1016/j.cosust.2013.07.001

Mellafe, R., Rebolledo, A., y Cárdenas, M. (1992). Historia de la Universidad de Chile. Santiago, Chile: Ediciones de la Universidad de Chile.

Ministerio de Educación. (1997). Marco de politica para la educación superior. Santiago, Chile: Ministerio de Educación.

Mollis, M. (2006). Latin American university transformation of the 1990s: Altered identities? En J. J. F. Forest \& P. G. Altbach (Eds.), International handbook of higher education (Vol. 18) (pp. 503-515). Dordrecht, Holanda: Springer.

Nicolescu, B. (2010). Methodology of Transhisciphinarity-Levels of Reality, Logic of the Included Middle and Complexity. Transdisciplinary Journal of Engineering \& Science, 1, 19-38. https://doi.org/10.22545/2010/0009

O’Brien, K., Reams, J., Caspari, A., Dugmore, A., Faghihimani, M., Fazey, I., .. Raivio. (2013). You say you want a revolution? Transforming education and capacity building in response to global change. Environmental Science \& Policy, 28, 48-59. https://doi.org/10.1016/j.envsci.2012.11.011

Olssen, M. (2015). Neoliberal competition in higher education today: Research, accountability and impact. British Journal of Sociology of Education, 37(1), 129-148. https://gi.org/10.1080/01425692.2015.1100530

Organización para la Cooperación y Desarrollo Económicos (OCDE) (2017). Principales indicadores de ciencia y tecnología y Estudios Económicos de la OECD. Santiago, Chile: OCDE.

Peters, M. (1992). Performance and accountability in 'post-industrial society's: The crisis of British universities. Studies in Higher Eduction, 17(2), 123-139. https://doi.org/10.1080/03075079212331382617

Pineda, P. (2014). Science and higher education policy and scientific inquiry: Chilean and Colombian private universities compared. Comparative \& International Higher Education, 6(Spring), 8-11. Recuperado de https://www.ojed.org/index.php/jcihe/article/view/850

Pineda, P. (2015). The entrepreneurial research university in Latin America: Global and local models in Chile and Colombia, 1950-2015. Nueva York, NY: Palgrave Macmillan US.

Powell, W. W. \& Snellman, K. (2004). The Knowledge Economy. Annual Review of Sociology, 30(1), 199-220. https://doi.org/10.1146/annurev.soc.29.010202.100037

Programa Regional de Investigación Científica y Tecnológica. (2018). Guia de instrumentos de Conicyt para Regiones 2018. Santiago, Chile: CONICYT.

Ramirez, F. (2006). Growing commonalities and persistent differences in higher education: Universities between global models and national legacies. En H. D. Meyer \& B. Rowan (Eds.), The New Institutionalism in Education (pp. 123-141). Albany, NY: State University of New York Press.

Rosenfield, P. (1992). The potential of transdisciplinary research for sustaining and extending linkages between the health and social sciences. Social Science \& Medicine, 35(11), 1343-1357. https://doi.org/10.1016/0277-9536(92)90038-R

Ruiz Schneider, C. (2010). De la república al mercado. Ideas educacionales y politica en Chile. Santiago, Chile: LOM Ediciones.

Russell, A. W., Wickson, F., \& Carew, A. L. (2008). Transdisciplinarity: Context, contradictions and capacity. Futures, 40(5), 460-472. https://doi.org/10.1016/j.futures.2007.10.005

Said, E. W. (1983). The world, the text, and the critic. Cambrigge, MA: Harvard University Press.

Salazar, J. y Leihy, P. S. (2013). El manual invisible: Tres décadas de políticas de educación superior en Chile (1980-2010). Archivos Analiticos de Politicas Educativas, 21, 1-35. https://doi.org/10.14507/epaa.v21n34.2013

Schofer, E. \& Meyer, J. W. (2005). The worldwide expansion of higher education in the twentieth century. American Sociological Review, 70(6), 898-920. https://doi.org/10.1177/000312240507000602

Scholy, R. (2017). The normative dimension in transdisciplinarity, transition management, and transformation sciences: New roles of science and universities in sustainable transitioning. Sustainability, 9(6), 991. https://doi.org/10.3390/su9060991

Serrano, S. (1994). Universidad y nación: Chile en el siglo XIX. Santiago, Chile: Editorial Universitaria.

Slaughter, S. \& Leslie, L. L. (1997). Academic capitalism: Politics, policies, and the entrepreneurial university. Baltimore, MD: Johns Hopkins University Press. 
Slaughter, S. \& Leslie, L. L. (2001). Expanding and elaborating the concept of academic capitalism. Organization, 8(2), 154-161. https://doi.org/10.1177/1350508401082003

Slaughter, S. \& Rhoades, G. (2004). Academic capitalism and the new economy: Markets, state, and higher education. Baltimore, MD: Johns Hopkins University Press.

Star, S. L. \& Griesemer, J. (1989). Institutional Ecology, “Translations” and Boundary Objects: Amateurs and Professionals in Berkeley's Museum of Vertebrate Zoology, 1907-39. Social Studies of Science, 19(3), 387-420. https://doi.org/10.1177/030631289019003001

Steinbicker, J. (2011). Zur Theorie der Informationsgesellschaft: Ein Vergleich der Ansätze von Peter Drucker, Daniel Bell und Manuel Castells (2a edición). Wiesbaden, Alemania: VS Verl.

Stokols, D., Fuqua, J., Gress, J., Harvey, R., Phillips, K., Baezconde-Garbanati, L., .. Trochim, W. (2003). Evaluating transdisciplinary science. Nicotine and Tobacco Research, 5(SUPPL. 1), S21-S39. https://doi.org/10.1080/14622200310001625555

Tünnerman, C. (2003). La universidad latinoamericana ante los retos del siglo XXI. Ciudad de México, México: Unión de Universidades de América Latina.

Universidad de Chile (2006). Proyecto de Desarrollo Institucional: El Compromiso de la Universidad de Chile con el Pais. Santiago, Chile: Universidad de Chile.

Universidad de Chile. (2018). Plan de Desarrollo Institucional de la Universidad de Chile 2017 - 2026. Santiago, Chile: Universidad de Chile.

Urquiza, A., Amigo, C., Billi, M., Brandão, G., y Morales, B. (2018). Metálogo como herramienta de colaboración transdisciplinaria. Cinta de Moebio: Revista Electrónica de Epistemología de Ciencias Sociales, 62, 182-198. https://doi.org/10.4067/S0717-554X2018000200182

Wang, J., Aenis, T., \& Siew, T. F. (2019). Communication processes in intercultural transdisciplinary research: framework from a group perspective. Sustainability Science, 14, 1673-1684. https://doi.org/10.1007/s11625-019-00661-4

Yarime, M., Trencher, G., Mino, T., Scholz, R. W., Olsson, L., Ness, B., ... Rotmans. (2012). Establishing sustainability science in higher education institutions: towards an integration of academic development, institutionalization, and stakeholder collaborations. Sustainability Science, 7, 101-113. https://doi.org/10.1007/s11625-012-0157-5 